\title{
Eyeblink Artifact Suppression from EEG Signal using Lifting Wavelet Transform
}

\author{
Mst. Jannatul Ferdous ${ }^{1,2, *}$, Md. Sujan $\mathrm{Ali}^{1,2}$, Md. Ekramul Hamid ${ }^{1}$ and \\ Md. Khademul Islam Molla ${ }^{1}$ \\ ${ }^{I}$ Signal Processing and Computational Neuroscience Lab, Department of Computer Science and Engineering, \\ University of Rajshahi, Rajshahi, Bangladesh \\ ${ }^{2}$ Department of Computer Science and Engineering, Jatiya Kabi Kazi Nazrul Islam University, Mymensingh, \\ Bangladesh \\ *E-mail: miferdous_cse@jkkniu.edu.bd
}

\begin{abstract}
In this paper we proposed a technique to remove eye blink artifact from electroencephalogram (EEG) using lifting wavelet transform (LWT). The LWT has been successfully used in eye blink artifact suppression form the recorded electroencephalography (EEG) signals using a data-adaptive subband filtering approach. The LWT is applied to decompose EEG signal into a finite set of subbands. The energy based subband filtering is implemented to separate the lower frequency noise components to clean the EEG signal. The energies of individual subbands respectively for EEG and $f G n$ that of contaminated EEG are compared to derive the energy based threshold for the suppression of eyeblink effects. We adopt two de-noising algorithms based on stationary subspace analysis (SSA), and lifting wavelet transform (LWT) for comparison purpose. Through using contaminated EEG signals from BCI database, we evaluate the artifact correction results by means of SAR and MSE, and conclude that LWT algorithm is the suitable one for de-noising EEG signal.
\end{abstract}

Keywords: electroencephalography, artifact reduction, stationary subspace analysis, lifting wavelet transform

\section{Introduction}

Brain-computer interface (BCI) has been one of the most outstanding biomedical engineering research fields for decades. It provides a promising technology allowing humans to control external devices by modulating their brain waves.The quality of electroencephalography (EEG) is degraded by the non-cerebral signal sources are known as artifacts. Among all the artifacts, ocular artifacts are the most prevailing. Ocular artifacts occur through eye movements and blink which generates a signal greater in magnitude than EEG signals, allowing it to travel throughout the scalp, covering and distorting EEG signals [1-4]. In order to achieve higher quality EEG signals, these artifacts must be removed with-out distorting or removing any of the underlying EEG data. There are various methods to control ocular artifacts. One of the modest methods is confining the eye movements and eye blinking of the subject by keeping on a fixed point. Though it is difficult for the subject to perform this task especially for infants or persons with certain disabilities during experiments. Moreover, the effort of performing the task can have a significant effect on the attained EEG data [5].

In previous research, many simple and complex methods have been proposed for detecting and removing artifacts. The simple signal processing filter, known as Butterworth bandpass filter is used to remove the artifact. The clean EEG signal is easily achieved from raw EEG by applying $4^{\text {th }}$ order of bandpass Butterworth filter. However, this type of filter does not suitable for EEG signal processing because some of the original information are loss during the filtration process [18]. ICA has become a popular method in removing artifacts from EEG data. The disadvantage of ICA is that the components do not necessarily only contain artifact data, but also contains underlying EEG data [2]. Removing the contaminated component will thus lead to loss of EEG data.

To address the weakness of ICA-based artifact correction methods another effective BSS method, stationary subspace analysis (SSA) is used [6,7]. The EOG signals are considered as low frequency and high energy trend in the recorded EEG signals, the SSA is performed better to suppress it from mixing. The components that are determined to be related to the ocular artifacts are projected back to be subtracted from EEG signals, producing the clean EEG data eventually. Hence it is very evident to employ SSA for eyeblink artifact identification and suppression. It is used for multiple channel data to partition the whole space into stationary and non-stationary subspaces [8].

The discrete wavelet transform has been extensively studied over the past 20 years. Many applications have been proposed but the power of wavelet transform lies in its performance in compression and denoising schemes. The existence of fast algorithms for its computation is another important factor. The well-known 
Mallat's algorithm (DWT- Discrete Wavelet Transform) employs two-channel filter bank iteratively and the filter bank can be equally represented by a polyphase lifting scheme. Iterative application of the lifting scheme (LWT-Lifting Wavelet Transform) results in the same coefficients as the DWT does. The lifting scheme was rediscovered by Sweldens in [9] and according to [10], every wavelet filter bank can be decomposed into a lifting scheme.

It is presented that the realization of a lifting wavelet processor which is used an integer type Haar lifting wavelet transform (LWT) to measure ECG signals in real time [11]. It is also adopted Db4 lifting wavelet transform to reconstruct ECG signals [12]. It is also proposed a lifting-based discrete wavelet transform to denoise ECG signals. Through testing with Haar, Db4, Db6, Filter (9-7), and Cubic B-splines, Db4 decomposition in 4 scales with level dependent threshold estimator can get best SNR and visual inspection in de-noising [13].

In this paper, lifting wavelet transform (LWT) approach is introduced to separate low frequency artifacts from contaminated EEG. The signal is decomposed using wavelet transform yielding the subbands. The individual subband is thresholded and added together to obtain the clean signal. Also, this paper aims to shows the comparison when EEG signal filter with lifting wavelet transform (LWT) and stationary subspace analysis (SSA). The comparative study presented that LWT approach is more effective to extract the EEG signals after suppressing the contaminated artifacts.

The remaining parts of the paper are organized as- the algorithms used in the paper (SSA, and LWT) are described in Section II, the experimental result and discussion of the artificially contaminated EEG data and performance metrics are explained in Section III, and finally Section IV gives a brief conclusion to the paper.

\section{Methods}

The recorded EEG is modeled as linear combination of pure EEG and EOG artifact defined as:

$$
S_{E E G}(t)=P_{E E G}(t)+A_{E O G}(t)
$$

where $S_{E E G}(t), P_{E E G}(t)$, and $A_{E O G}(t)$ are measured EEG, pure EEG and EOG artifact respectively. The measured EEG signal is the superposition of original EEG signal due to brain activity and the fraction of EOG signal due to eye blink activity. The aim of the proposed scheme is to extract required EEG signal $P_{E E G}(t)$ from the measured signal $S_{E E G}(t)$ which consists of the required signal $P_{E E G}(t)$ plus the ocular artifact signal $A_{E O G}(t)$. In this paper, multichannel $S_{E E G}(t)$ is used as the primary input to suppress its EOG artifact using SSA and wavelet based techniques. No reference channel is used to estimate the pure EEG signals.

\section{A. Stationary Subspace Analysis}

Stationary Subspace Analysis (SSA) is a blind source separation algorithm which factorizes a multivariate time series into stationary and non-stationary components. In EEG analysis, the electrodes on the scalp record the activity of a large number of sources located inside the brain. These sources can be stationary or non-stationary, but they are not discernible in the electrode signals, which are a mixture of these sources. SSA allows the separation of the stationary from the non-stationary sources in an observed time series.

According to the SSA model [8], the observed multivariate time series $\mathrm{s}(\mathrm{t})$ is assumed to be generated as a linear superposition of stationary sources $s^{s}(t)$ and non-stationary sources $s^{n}(t)$ as follows:

$$
s(t)=M n(t)=\left[\begin{array}{ll}
M_{s} & M_{n}
\end{array}\right]\left[\begin{array}{l}
s^{s}(t) \\
s^{n}(t)
\end{array}\right]
$$

where, $M$ is an unknown but time-constant mixing matrix; $M_{s}$ and $M_{n s}$ are the basis of the stationary and nonstationary subspace respectively. Given samples from the time series $s(t)$ the aim of SSA is to estimate the inverse mixing matrix $M^{-1}$ separating the stationary from non-stationary sources in the mixture.

Using the estimated mixing matrix, $\hat{M}=\left[\begin{array}{ll}\hat{M}_{s} & \hat{M}_{n}\end{array}\right]$ given by SSA, these artifactual components $X_{\text {art }}(t)$ are projected back to EEG channel, and artifacts in EEG data $S_{\text {art }}(t)$ are calculated as

$$
s_{\text {art }}(t)=\hat{M}_{n} X_{a r t}(t)
$$


Finally, this ocular activity is removed from the EEG recording to yield the clean EEG data $\hat{s}(t)$ by the following formula:

$$
\hat{s}(t)=s(t)-s_{a r t}(t)
$$

where, $\hat{s}(t) \approx S_{E E G}(t)$. Thus the pure EEG of any channel is reconstructed using Eq. (2).Using the above calculating formula we get the clean EEG from artificially contaminated EEG and the process is depicted in Fig. 1.

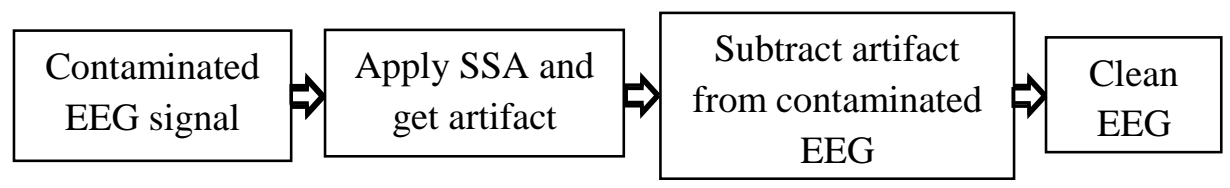

Fig. 1. Block diagram of stationary subspace analysis approach.
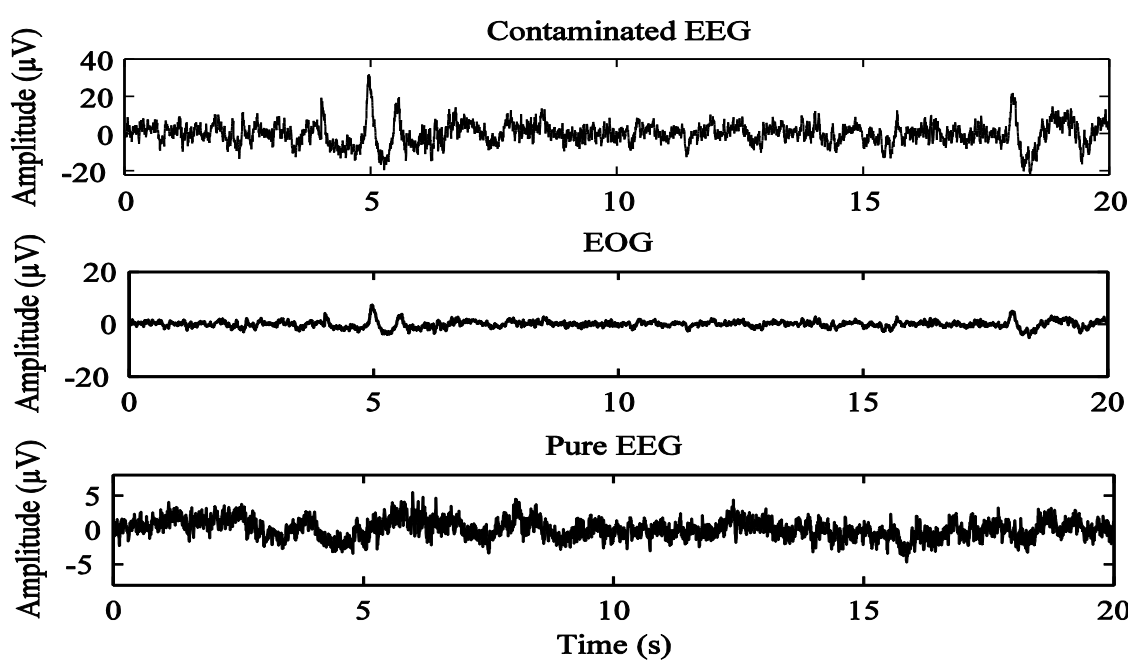

Fig. 2. The separation of clean EEG from the contaminated data using SSA.

The EOG suppression results for a single channel (multichannel is used for SSA experiment) of recorded electroencephalography are illustrated in Fig.2. in which the separated electro-oculogram and purified EEG signals are shown in the second and third rows respectively.

\section{B. Lifting Wavelet Transform}

Lifting scheme was introduced by Sweldens in 1995 had simplified the mechanism in constructing wavelets and this approach becomes more practical to be realized for real-time applications $[9,10,14,15]$. LWT reduces to the polyphone version of the DWT algorithm with zero-padding extension mode and without extracoefficients. The scheme does not require the information in Fourier transform because the wavelet transform can be implemented in spatial or time domain. The basic steps in lifting operations are:

1) Split: The original signal of length $\mathrm{n}$ where $n=2 \mathrm{j}$ is separated into two disjoint sets of even and odd samples.

2) Predict: The predict step replaces the odd element with this difference as in Eq. (3). and can be considered as high frequency or detail components. Therefore the predict step can be viewed as high-pass filter. This is done by the following equation:

$$
d_{j-1}[n]=x_{o}[n]-p\left(x_{e}[n]\right)
$$

where $p$ is the predict operator.

3) Update: This step replaces the even element with an approximation that is the signal becomes smoother compare to the previous scale. Hence this operation is viewed as low-pass filtering since the smoother signal contains fewer high frequency components. The update equation is as follows:

$$
a_{j-1}[n]=x_{e}[n]+u\left(d_{j-1}[n]\right)
$$

where $u$ is the update operator.

4) Normalization: The approximation and details coefficients must be normalized in the final step of the transformation. 


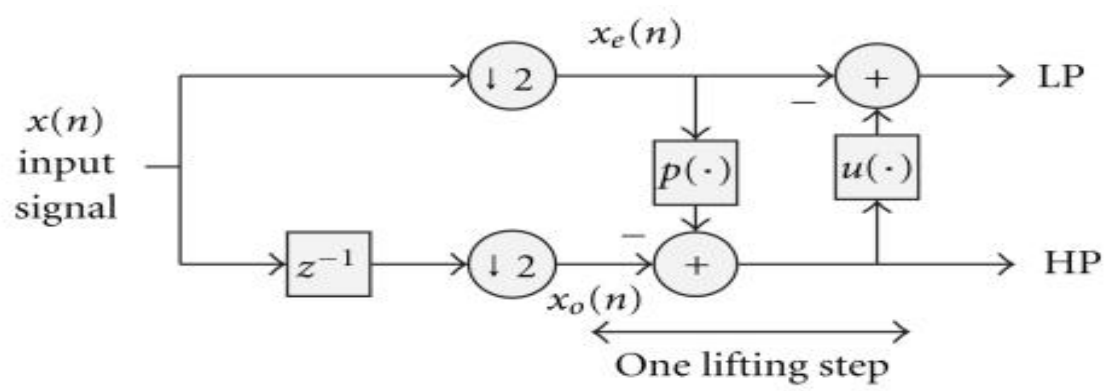

(a)

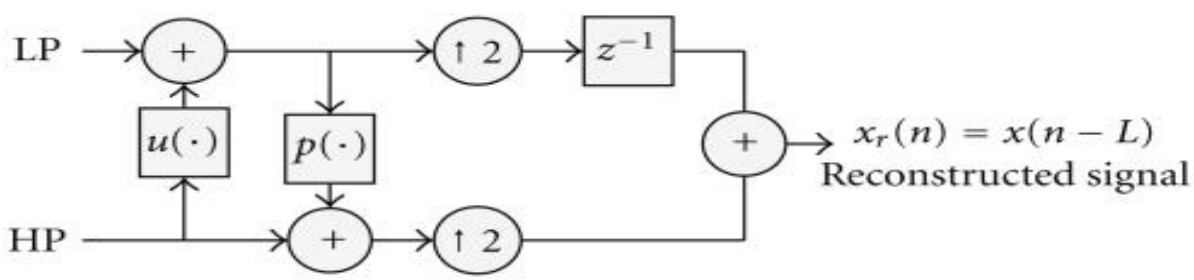

(b)

Fig. 3. (a)One step forward lifting operation. (b) Inverse lifting step

The lifting step is depicted in Fig.3.(a) for the decomposition or analysis of the forward wavelet transform. The update and predict stages can become a pair but sometimes they may not be together in a lifting step.

To obtain the signal back, the operations can be undone by just reversing them and change the signs as shown in Fig. 3.(b). The operation is working backwards from the forward lifting operation.

If more steps are required, they can be added singly. The first generation wavelets can be converted into lifting steps by factoring its h-coefficients as shown in many texts $[9,10,14,15]$.In this study, a noise assisted LWT based approach is implemented here to reduce the low frequency noise from single channel EEG. At the end of the decomposition, the reconstructed signal is represented as

$$
\tilde{s}(t)=\sum_{b=1}^{L+1} q_{b}(t)
$$

where, $\tilde{s}(t) \approx \mathrm{x}(\mathrm{t})$. The LWT based filtering method of EEG signal is shown in Fig. 4. In this Fig., the clean EEG signal is easily achieved from raw EEG by applying LWT .Then the clean EEG is evaluate by the same evaluation parameter used in SSA. The db4 mother wavelet is choosing for this filter. The lower frequency EEG signal of the single channel can be estimated by summing up the lower order subbands as:

$$
\hat{s}(t)=\sum_{b=1}^{D} C_{b}(t)
$$

where, $C_{b}(\mathrm{t})$ is the $\mathrm{b}^{\text {th }}$ subband of the channel. Here, the subject is to find the critical (threshold) subband with index $\mathrm{D}$ such that the subbands of indices $1,2,3, \ldots, \mathrm{D}$ are responsible for relatively lower frequency pure EEG component.

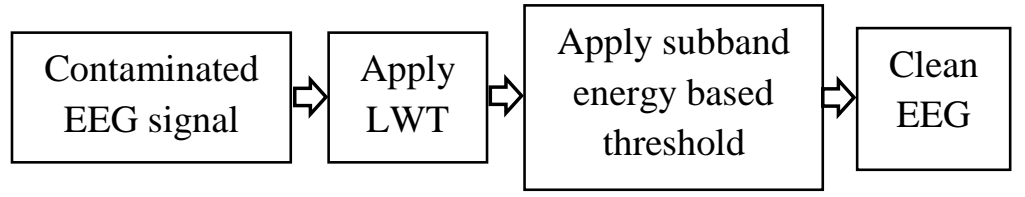

Fig. 4. Block diagram of lifting wavelet transform approach.

It is a well-recognized wavelet transform of a 1-D signal and inverts the transform to demonstrate perfect reconstruction. The original signal, reconstructed signal using LWT method and the reconstruction error are illustrated in Fig.6. 


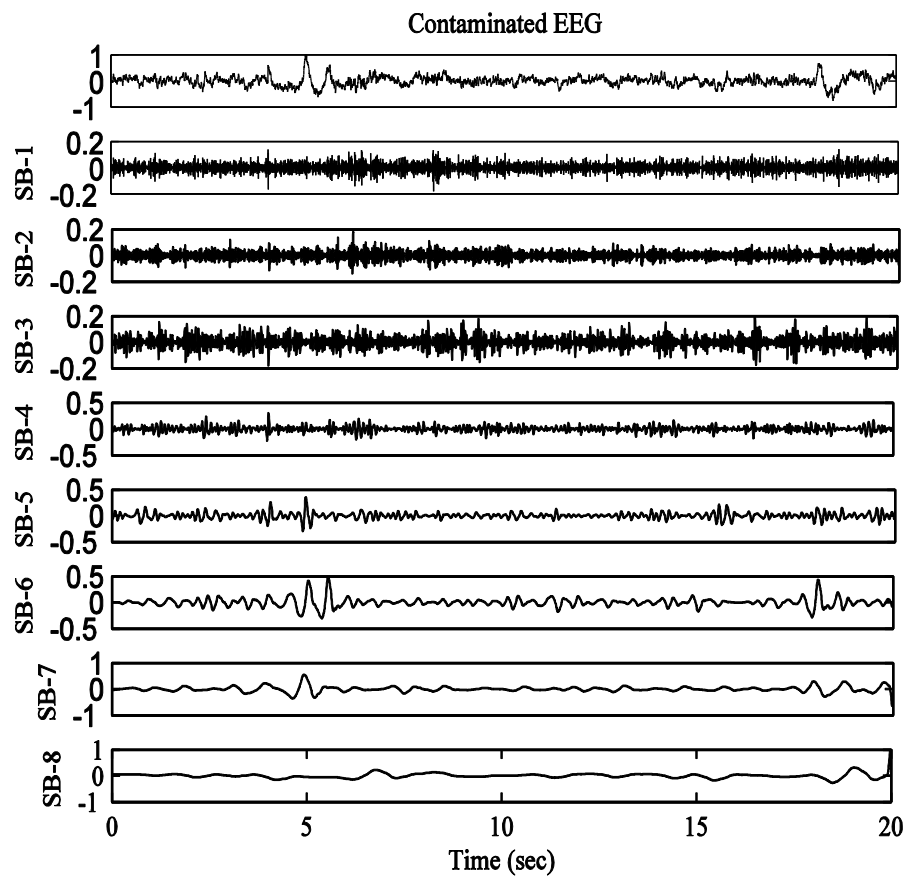

Fig. 5. The subband decomposition of contaminated EEG using LWT.

\section{Results and Discussion}

In this section, the LWT based method is tested with the artificially corrupted EEG signal and after that the signal is comprised with SSA. For comparison purposes, the time series contaminated EEG data is decomposed into multiple subbands using SSA and lifting wavelet transforms (LWT).

The SSA is used for multiple channel data and its decomposition is produced stationary and nonstationary components. More precisely, the target EEG is considered as stationary with respect to artifacts of higher order non-stationary. To get the desired clean EEG data, the artifactual component is subtracted from contaminated EEG [8]. The experimental result for the first channel is illustrated in Fig.2. It is observed that the purified EEG signal holds some artifact and the EOG is not separated properly but includes some signal components of EEG signal.

Another potential multiresolution decomposition approach, LWT which is analyzing a non-stationary signal like EEG. In this study, "db4" is used with 7 level decomposition. It is another approach for EOG artifact reduction. To get artifact free EEG the LWT based filter is used. The raw EEG signal and a reference signal fGn are decomposed up to level 7 using db4 mother wavelet. After decompose, we got 8 subbands. The subband decomposition of recorded electroencephalography data and fractional Gaussian noise are shown in Fig. 5 and Fig. 7 respectively. The algorithm for LWT to find the index D of the threshold subband for the channel is following:

1) Decompose the analyzing EEG signal together with the fGn ( in Fig.7.) into a finite set of subbands using LWT.

2) Calculate the energies of the subbands of fGn and its $95 \%$ confidence interval (CI).

3) Compute the energies of all subbands of contaminated EEG. Then find the lowest order subband with energy exceeding the upper limit of CI derived in step 2 say it is the $n$th subband. The selected $n^{\text {th }}$ (in Fig.8(a), $n=5$ ) subband is the starting index to reconstruct electro-oculogram signal.

4) The EEG is separated by summing up the subband coefficient starting from 1 to $D^{\text {th }}$ subband of electroencephalography signals using Eq. (6).

The determination of threshold subband based on the subband energy is illustrated in Fig.8.(a). After computing the index D of threshold subband (for EEG channel), the pure EEG of that channel is separated using Eq. (6). 

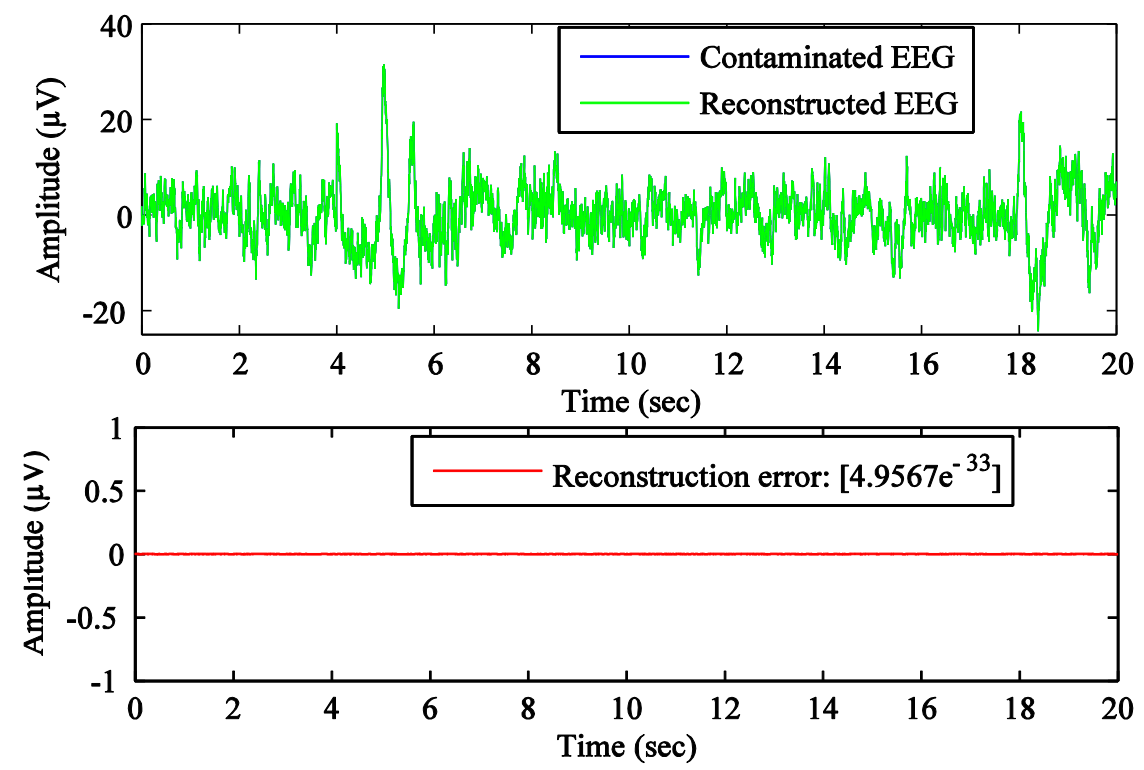

Fig.6. Reconstruction of contaminated EEG (top) and reconstruction error (bottom) using LWT.

It is observed in Fig. 8.(a) that the $5^{\text {th }}$ subband is the first subband index that exceeds the upper limit of confidence interval and the total number of subbands are 8 . The $5^{\text {th }}$ subband coefficient is the starting point of lower frequency components. The electro-oculogram is separated by summing the subband coefficients 5 to 8 . By subtracting electro-oculogram from raw electroencephalography, we get the purified electroencephalography that reflects the actual neural activities. The electro-oculogram suppression results for a single channel of recorded electroencephalography are illustrated in Fig.8.(b) in which the separated electro-oculogram and purified electroencephalography signals are shown in the second and third rows respectively. From Fig. 8.(b), it is observed that the purified EEG signal contains more original information although the artifact has cancel out. It is apparent that using SSA for artifact correction, underlying EEG or low frequency cerebral data may be lost. In order to reduce the data loss LWT method is used.

Fig. 9. depict the contaminated EEG and clean EEG for the two methods. From this Fig., it is observed that the LWT baesd method is best for reduce the EOG from contaminated EEG without cutting the information and assist to get clean EEG. The pure EEG do not show any large EOG.As a result, the purified EEG signal is found as completely artifact free in LWT method.

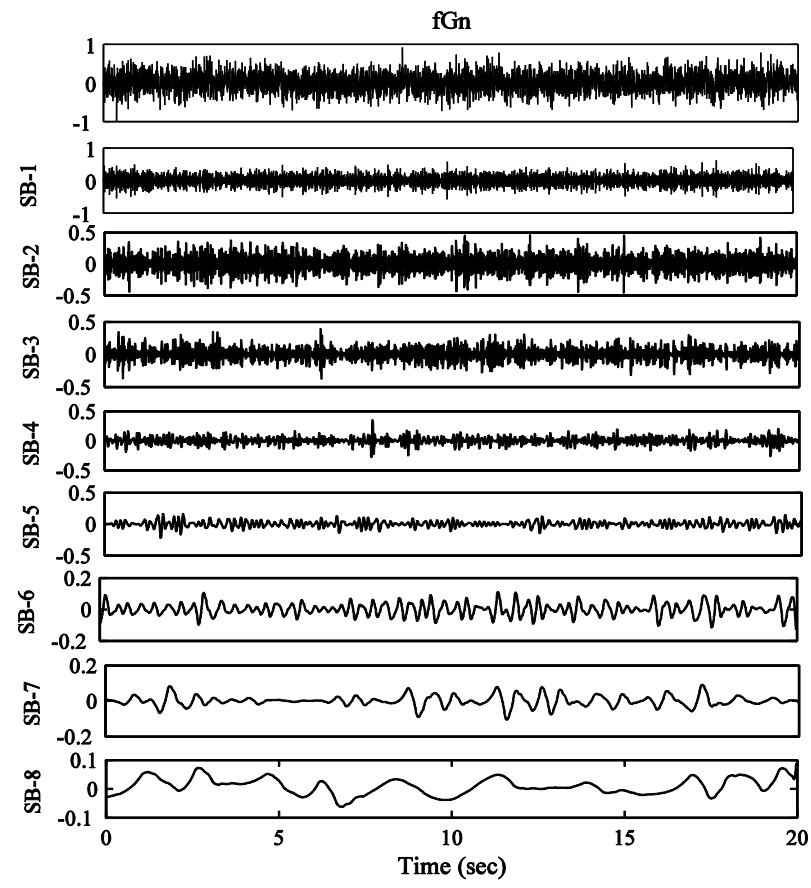

Fig. 7. The subband decomposition of fGn using LWT. 
Performance metrics:

In order to determine whether the method is successful at removing ocular artifact (OA) from EEG, the performance is assessed using two statistical parameters i.e. signal to artifact ratio (SAR) and mean square error (MSE).

Signal to artifact ratio (SAR):

The metrics commonly employed to represent the energy of the signal compared to the energy of the artifact is the signal to artifact ratio (SAR) [16, 17].

$S A R_{d B_{\text {contaminated_EEG }}}=20 \log 10 \frac{r m s[x(t)]}{r m s[s(t)-\hat{s}(t)]}$

Here, $x(t)$ is the clean EEG signal. $s(t)$ stands for contaminated EEG signal, $\hat{s}(t)$ is artifact free EEG signal and $\mathrm{N}$ for the signal length or the number of samples.

Mean square error (MSE):

It is used to describe similarity between the original signal and de-noised signal.

$M S E=\frac{1}{N} \sum_{i=1}^{N}\left[s_{i}(t)-\hat{s}_{i}(t)\right]^{2}$

$s_{i}(t)$ stands for original signal, $\hat{s}_{i}(t)$ estimates signal de-noised by LWT, $\mathrm{N}$ for the signal length or the number of samples.

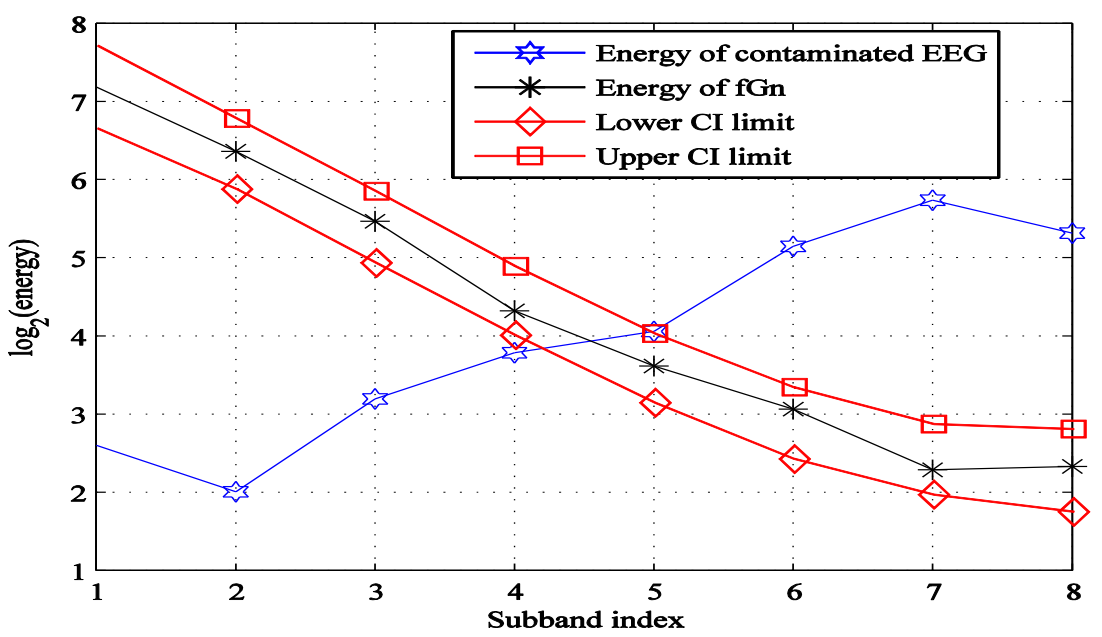

Fig.8.(a) The selection of threshold from subband energy of contaminated EEG channel (by applying LWT) based on the subband energy of fGn.
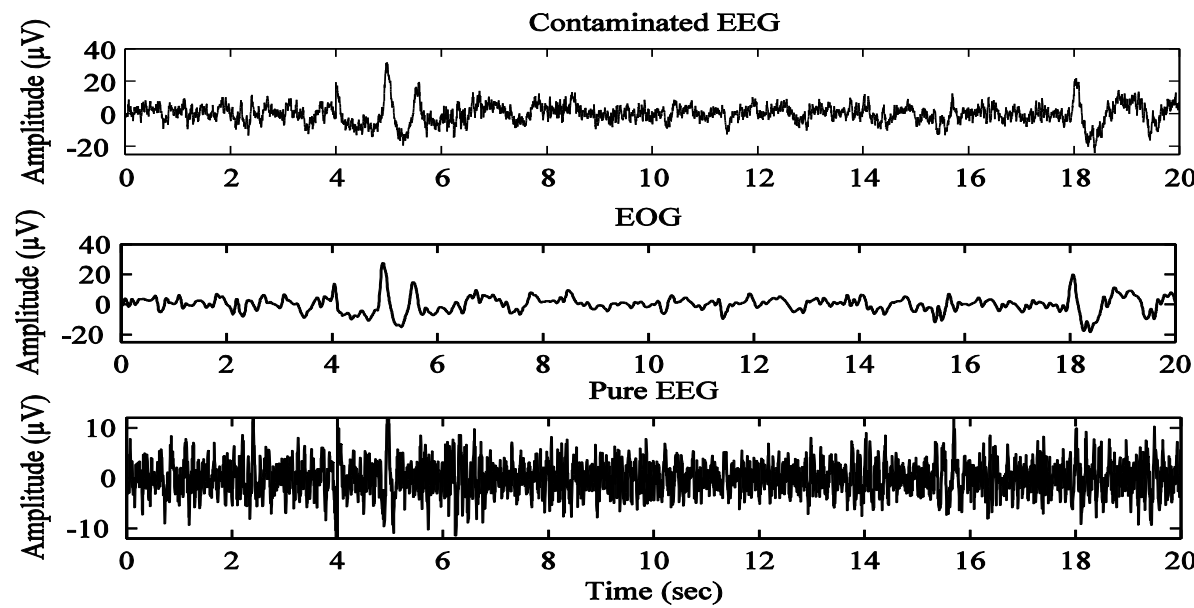

Fig.8.(b) Mixture of eyeblink like contaminated EEG signal and pure EEG. (Top): contaminated EEG signal. (Middle): EOG signal. (Bottom): the pure EEG signal using LWT. 


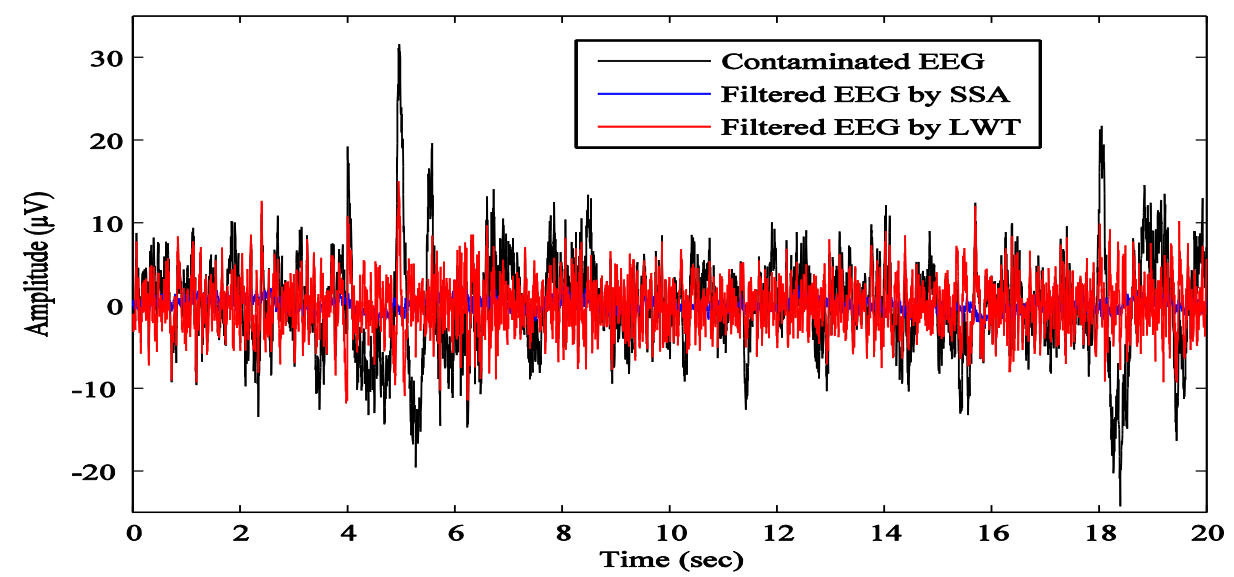

Fig. 9. Visual comparison of the simulation results corresponding to the contaminated EEG signal (i.e. mixedup of EEG and EOG signal), and the corrected EEG signals after applying different ocular artifact removal methods.

TABLE I. A comparative summary of the stationary subspace analysis and lifting wavelet transform for contaminated EEG data.

\begin{tabular}{|l|l|l|l|}
\hline No. & Methods & SAR in dB & MSE \\
\hline 1. & SSA & -6.04 & 12.15 \\
\hline 2. & LWT & -4.70 & 10.19 \\
\hline
\end{tabular}

TABLE I shows the comparison results of the SAR and MSE values from stationary subspace analysis and LWT methods, respectively. The table shows that the LWT based technique yields the best SAR (lower the SAR value able to clean more artifact) result than SSA.The SAR value is $-6.04 \mathrm{~dB}$ for stationary subspace analysis while for LWT filter it is $-4.70 \mathrm{~dB}$. The lifting wavelet transform shows lower MSE value compared to the SSA. Based on the MSE and SAR values, it is observed that the lifting wavelet transform filter with db4 mother wavelet is able to filter out more noise compare to stationary subspace analysis.

\section{Conclusions}

It is very important to filter the artifact before analyze EEG data. EEG signal is contaminate by various artifacts. In this paper, two different artifact removing techniques have been presented to determine their effect in removing the artifact. We have successfully implemented the scheme of eyeblink artifact suppression from contaminated electro- encephalography signals using SSA and LWT. The electro-oculogram signal is considered as the trend of the recorded electroencephalography signals. The trend is detected by comparing the energy of individual subband energy with that of the reference signals i.e. fractional Gaussian noise in LWT filter. The MSE and SAR is a popular parameter to determine the quality of signal after filtering. LWT filter able to remove electrooculography artifacts while the stationary subspace analysis does not able to remove all of artifact. The lifting wavelet transform is better in removing the noise compare to of the stationary subspace analysis.

\section{Acknowledgements}

This research work is supported by the Information and Communication Technology (ICT) division of the ministry of Post, Telecommunication and Information Technology, Bangladesh.

\section{Conflicts of Interest}

The authors declare that there is no conflict of interests regarding the publication of this paper.

\section{References}

[1] S. V. Ramanan, N. V. Kalpakam, J. S. Sahambi, "A novel wavelet based technique for detection and de-noising of ocular artifact in normal and epileptic electroen-cephalogram", ICCCAS, pp. 180-183,27-29 June 2004.

[2] C. Joyce, I. Gorodnitsky, M. Kutas, "Automatic removal of eye movement and blink artifacts from EEG data using blind component separation", Psychophysi-ology 41, 313-325, 2004.

[3] V. Krishnaveni, S. Jayaraman, S. Aravind, V. Hariharasudhan, K. Ramadoss, "Auto-matic identification and removal of ocular artifacts from EEG using wavelet transform", Meas. Sci. Rev. 6 (4) ,45-57, 2006.

[4] Z. Wang, P. Xu, T. Liu, Y. Tian, X. Lei, D. Yao, "Robust removal of ocular arti-facts by combining independent component analysis and system identification”, Biomed. Signal Process. Control 10, 250-259, March, 2014.

[5] M. Garcia, M. Thomlinson, J. Lopez, B. Jervis, C. Mair, "Residual ocular arte-fact subsequent to ocular artefact removal from the electroencephalogram”, IEEProc. - Sci. Meas. Technol. 146, 293-298, November, 1999. 
[6] P. V. Bunau, F. C. Meinecke, F. C. Kiraly, and K. R. Muller, "Finding stationary subspaces in multivariate time series," Physical Review Letters, vol. 103, no.21, Nov. 2009.

[7] S. Hara, Y. Kawahara, T. Washio, P. von Bunau, and T. Tokunaga, "Separation of stationary and non-stationary sources with a generalized eigenvalue problem," Neural Networks, vol. 33, pp.7-20, 2012.

[8] H. Zeng and A. Song, "Removal of EOG artifacts from EEG recordings using stationary subspace analysis," The Scientific World Journal, vol. 2014, pp. 1-9, 2014.

[9] W. Sweldens, "The lifting scheme: A construction of second generation wavelets," SIAM J. Math. Anal., vol. 29, no. 2, pp. 511546, 1997.

[10] I. Daubechies and W. Sweldens, "Factoring wavelet transforms into lifting steps," J. Fourier Anal. Appl., vol. 4, no. 3, pp. 245-267, 1998.

[11] Kuzume K, Niijima K, “Takano S, FPGA-based lifting wavelet processor for real-time signal detection,” SIGNAL PROCESSING, 2004, 84(10), pp. 1931-1940.

[12] Sai, R.Ben, Messaoud, M.,Kachouri,A.,Sellami, F., “Analysis of the electrocardiogram signal by the lifting scheme,” International Symposium on Control, Communications and Signal Processing,ISCCSP, pp. 263-266, 2004.

[13] Erelebi, E., "Electrocardiogram signals de-noising using lifting-based discrete wavelet transform," Computers in Biology and Medicine, 34 (6), pp. 479-493, 2004.

[14] W. Sweldens and P. Schroder, "Building your own wavelets at home,"Wavelets in Computer Graphics, ACM Siggraph Course Notes, pp. 15-87. 1996.

[15] A. Jensen and la Cour-Harbo, “A. ripples in mathematics: discrete wavelet transform,” Germany: Springer-Verlag, 2001.

[16] S. Romero, M. A. Mañanas, and M. J. Barbanoj, "A comparative study of automatic techniques for ocular artifact reduction in spontaneous EEG signals based on clinical target variables: a simulation case comput.” Biol. Med., vol.38, pp.348-60,2008.

[17] M. H. Soomro, N. Badruddin, M. Z. Yusoff, and A. S. Malik, "A method for automatic removal of eye blink artifacts from EEG based on EMD-ICA," in Proc. IEEE 9th Int. Colloq. Signal Process. Appl., pp. 129-134.March 2013.

[18] M. J. Ferdous, M.S. Ali, M. E. Hamid and M. K. I. Molla, "A comparison of butterworth bandpass filter and discrete wavelet transform filter for the suppression of ocular artifact from EEG signal”, International Journal of Research in Engineering Technology, vol. 1 Issue 4, May - June 2016. 